\title{
Robotic Parathyroidectomy, A Good Alternative - Our Perspective
}

\section{Kalpana Nagpal*, Noor Ul Din Malik, Gayathri Bhaskaran, and Punita Bhatia}

Indraprastha Apollo Hospital, India

*Corresponding Author: Kalpana Nagpal, Senior Consultant, Indraprastha Apollo Hospital, India.
Received: October 01, 2021

Published: December 30, 2021

(C) All rights are reserved by Kalpana Nagpal., et al.

\begin{abstract}
Parathyroid surgeries have gained a lot of popularity because of the immediate result and relief of symptoms in the patient. They are aimed at correcting the imbalance in calcium and phosphate homeostasis.. Parathyroid surgery have evolved over time from open surgeries involving the identification of glands under normal vision to minimally invasive techniques. With the advent of new technologies, parathyroid surgeries have become easier and less traumatic physically and psychologically. Patient has an easier recovery period and because the newer techniques involve a hidden scar, coametic results are great. We present a case of parathyroid adenoma which was managed by robotic parathyroidectomy.
\end{abstract}

Keywords: Parathyroid; Robotic Parathyroidectomy; Headache

\section{Introduction}

Parathyroid glands are endocrine glands secreting parathyroid hormone which play an important role in calcium homeostasis. Elevated levels of parathyroid hormones due to a pathology in the parathyroid glands results in the condition called primary hyperparathyroidism. Eighty percent of the cases of primary hyperparathyroidism arises from a single functioning parathyroid adenoma. Surgical removal (targeted approach) of the adenoma is the preferred and most effective management option.

\section{Case Presentation}

A 54 year old male patient presented to the physician with history of muscle cramps and on and off headache. He was evaluated and found to have a parathyroid hormone level of $76 \mathrm{pg} / \mathrm{ml}$ with serum calcium of $12.5 \mathrm{mg} / \mathrm{dl}$. CT scan showed bulky thyroid gland and a Tc-99m-sesta MIBI Scan revealed an increased uptake in the left side of neck and retention after washout in the posteroinferior part of thyroid gland in the normal anatomical position of the Inferior parathyroid. After complete evaluation, informed consent and pre-anaesthesia checkup, Once the patient was under general anaesthesia, the first intraoperative sample of parathyroid hormone was assayed and found to be $76 \mathrm{pg} / \mathrm{ml}$. Patient was positioned with neck extension, left side retroauricular hairline incision given. Flap was elevated. Dissection done in a subplatysmal plane anterior to the sternocleidomastoid. A vertical tunnel was created between the platysma and thyroid fossa. Strap muscles were identified and separated. Chung retractor placed for flap retraction and da Vinci robot docked in. Remaining strap muscles separated from the thyroid capsule using harmonic scalpel and left lobe of thyroid identified and rotated superomedially. The parathyroid gland along with the adenoma immediately identified because of its size and colour which otherwise would have taken time under normal vision. The brownish nodular tumor removed and sent for histopathological study. Blood sample was sent for parathyroid hormone 10 minutes after removal and found to be $25 \mathrm{pg} / \mathrm{ml}$ reduced to less than 50 percent of the preoperative value, indicating successful removal of the adenoma. Wound was then closed in layers after placing drain. Operating time was 20 minutes from docking to drain placement. Postoperative care was given, drain was found to be almost nil and hence removed and patient was fine with minimal postoperative pain, hence was discharged on day 2. Histopathology was suggestive of parathyroid adenoma 


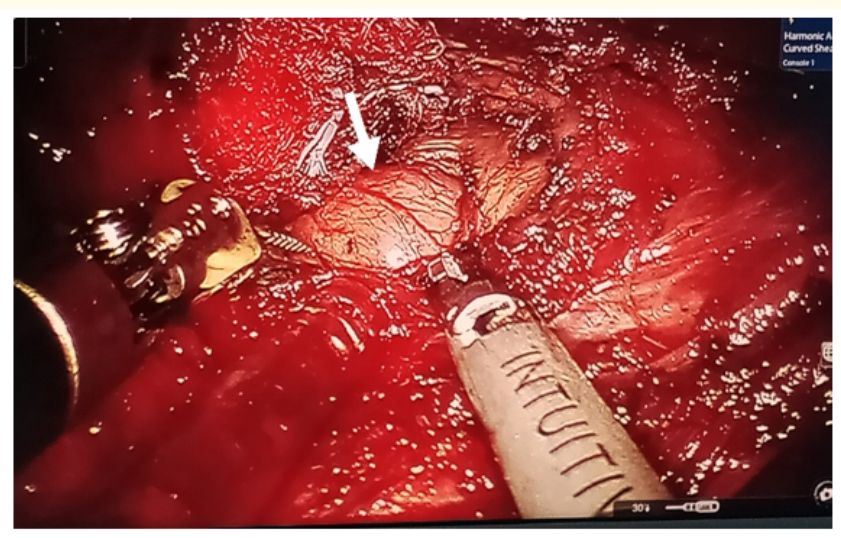

Figure 1: Left lobe of thyroid after dissection.
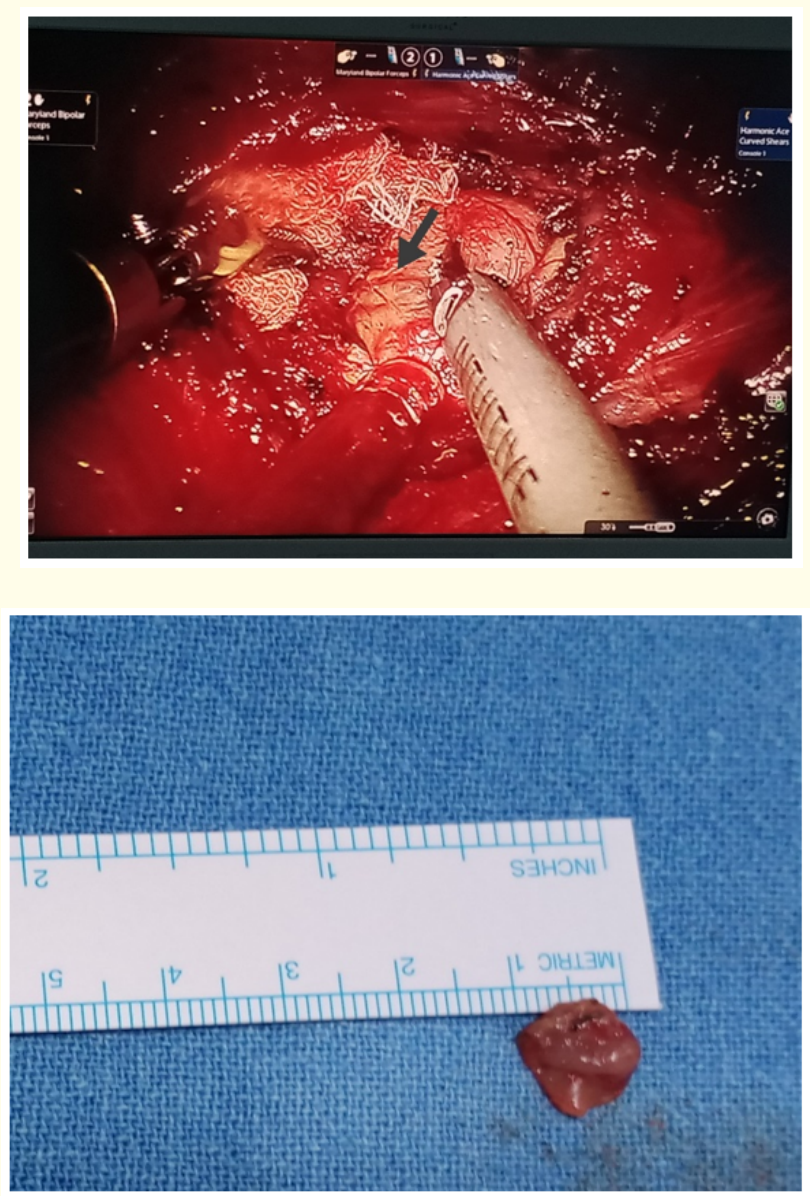

Figure 2: Left inferior parathyroid adenoma.

\section{Discussion}

\section{History}

The first successful parathyroid surgery by an open approach was done by Felix Mandel in 1925 [1]. Conventional explorative approach starting with a neck incision and dissection and exposure of all the four glands followed by removal of the grossly abnormal gland has been slowly replaced by the minimally invasive approach including endoscopic parathyroidectomy. Robotic parathyroidectomy has been considered as the $4^{\text {th }}$ generation in the evolution of parathyroid surgery as described by Tolley [2].

Procedure and work up

Blood investigations like serum calcium, phosphate and parathyroid hormone level and an ultrasound scan of the neck play an important role as screening techniques for hyperparathyroidism. Tc SestaMIBI scan is almost confirmatory and highly effective in identifying the location. Sensitivity and the positive predictive values of neck ultrasonography were $86.5 \%$ and $97.6 \%$ respectively and for Tc-99m-MIBI was $97.2 \%$ and $100.0 \%$ respectively. Intraoperative PTH assay (fall to less than 50 percent) according to Miami criteria is suggestive of complete excision of functional tumour [3].

\section{Advantages}

The da Vinci surgical robot offers three dimensional image with 10X magnification and depth perception. The robotic instruments possess seven degrees of freedom, motion scaling, and tremor filtering, thus contributing to excellent surgical precision and dexterity [2]. The proportion of patients interested in having a scarless surgery is quite high [4]. Eye tracking technology has been used to prove that scars draw attention from the face to neck [5]. Hence Robotic surgery scores in this aspect resulting in no visible scar as the scar is hidden in the hairline. Also a study of Dermatology Life Quality Index suggests that neck scars affect quality of life more than even keloids and psoriasis [6]. In our surgery, we have gone completely gasless, Hence side effects of insufflating with Carbon dioxide gas is not there. Since the magnification is high, very vital and minute structures like the parathyroid branch of the inferior thyroid artery were visualised and preserved. Moreover preservation of other structures like inferior thyroid artery and recurrent laryngeal nerve becomes easy. 
We prefer the Retroauricular approach than the transaxillary approach in view of risk of brachial plexus injury. Moreover tissue dissection and blood loss is relatively lower.

\section{Limitations}

Robotic parathyroid surgery is suitable only for selective patient profile including age, economic status and skin thickness and BMI. Moreover the cost-effectiveness cannot be commented upon because of current restriction to limited number of patients and relatively high cost involved. Awareness among patients and medical fraternity is currently lacking.

\section{Conclusion}

Robotic surgery is a great addition to a scarless generation of neck surgeries. Since it is still an evolving surgical practice, evidence is still at the grassroot level. Nevertheless, clear benefits are noticed with robotic assistance. Currently it is confined to highly equipped centres and experienced surgeons and select patients as the awareness is still lacking. Probably with the increasing production and usage, the cost of surgery can be expected to drop which takes care of the major disadvantage of cost-effectiveness.

\section{Bibliography}

1. Delbridge LW and Palazzo FF. "First parathyroid surgeon: Sir John Bland-Sutton and the parathyroids". ANZ Journal of Surgery 77 (2007): 1058-1061.

2. Tolley N., et al. "Robotic-assisted parathyroidectomy: a feasibility study". Otolaryngology-Head and Neck Surgery 144 (2011): 859-866.

3. Kandil E., et al. "Robotic-assisted parathyroidectomy via transaxillary approach: feasibility and learning curves". Gland Surgery 10.3 (2021): 953-960.

4. Wong W., et al. "Simplified minimally invasive parathyroidectomy: a series of 100 cases and review of the literature". Annals of The Royal College of Surgeons of England 93 (2011): 290293.

5. Juarez MC., et al. "Objectively measuring social attention of thyroid neck scars and transoral surgery using eye tracking". Laryngoscope 129 (2019): 2789-2794.

6. Choi Y., et al. "Impact of postthyroidectomy scar on the quality of life of thyroid cancer patients". Annals of Dermatology 26 (2014): 693-699.

\section{Assets from publication with us}

- Prompt Acknowledgement after receiving the article

- Thorough Double blinded peer review

- Rapid Publication

- Issue of Publication Certificate

- High visibility of your Published work

Website: www.actascientific.com/

Submit Article: www.actascientific.com/submission.php

Email us: editor@actascientific.com

Contact us: +919182824667 